\section{IN THE NEWS}

Peer review - the big secret?

Three quarters of the British public have no idea what peer review is, according to a new poll that was

commissioned by the

Science Media Centre and Nature.

The poll, conducted by the MORI Social Research Institute, involved interviewing more than a 1,000 adults aged 15 and over. The results were startling or unsurprising, depending on your point of view - only a quarter of those interviewed described peer review as "society's scrutiny of other scientists' work, generally" (BBC

Radio 4, Today programme). Intriguingly, however, the survey also showed that the public supports rigorous scrutiny of scientific results before publication, and if peer review did not exist already they would want to create it. "The vast majority $(71 \%)$ of the public favour either the kind of scrutiny provided by peer review or more stringent controls in which experiments are repeated independently before being published" (The Guardian). Fiona Fox, director of the Science Media Centre, encouraged the scientists to "get out there and share their big secret" of peer review.

These findings are of course timely - they were published only a few days after the "IVF specialist Dr Panos Zavos announced to the press that he had cloned a baby" having "refused to submit his experiment to peer review" (The Guardian). So, the poll's results seem to say that it is not only the scientists who are frustrated with this kind of science reporting, but that the public is weary as well.

There is a constructive outcome to this survey the Science Media Centre has published a new guide for scientists "in an effort to help them better communicate their work" (The Guardian).

Magdalena Skipper

\title{
Escape from Planet X
}

Genes on the mammalian X chromosome just can't wait to get off.

J. J. Emerson and colleagues' analysis of the human and mouse genomes shows that the $\mathrm{X}$ chromosome has a clear excess of genes that have functional duplicates on other chromosomes. Of the 94 genes that the authors identified to have been functionally retroposed between chromosomes in the human genome, 15 were derived from $\mathrm{X}$-chromosome genes: far more than the 3 or 4 expected on the basis of the size of this chromosome. Similarly, the 17 out of 105 functional retropositions in the mouse genome were of $\mathrm{X}$-chromosome genes, although only 4 or 5 were expected.

So why do genes want out of the $\mathrm{X}$ chromosome? One possibility is that genes that benefit males at a cost to females are moving because, compared with the X chromosome, an autosome spends on average less time in females and so would be more difficult to select against. Alternatively, the inactivation of $\mathrm{X}$-linked genes during meiosis might favour the export of genes to the autosomes, where they are more likely to be expressed to the benefit of the male during meiosis. Either of these mechanisms could cause functional retrogenes that are exported from the $\mathrm{X}$ to be selectively favoured over genes that are retroposed from other chromosomes.

Despite the X chromosome being a popular place for genes to leave, paradoxically, it also seems that it is a favourite destination. The authors show that there are relatively many more functional retrogenes recruited to the $\mathrm{X}$ than any other chromosome in both human and mouse genomes. However, they also show that human pseudoretrogenes, which are less likely to be subject to selection, are also more common than expected on

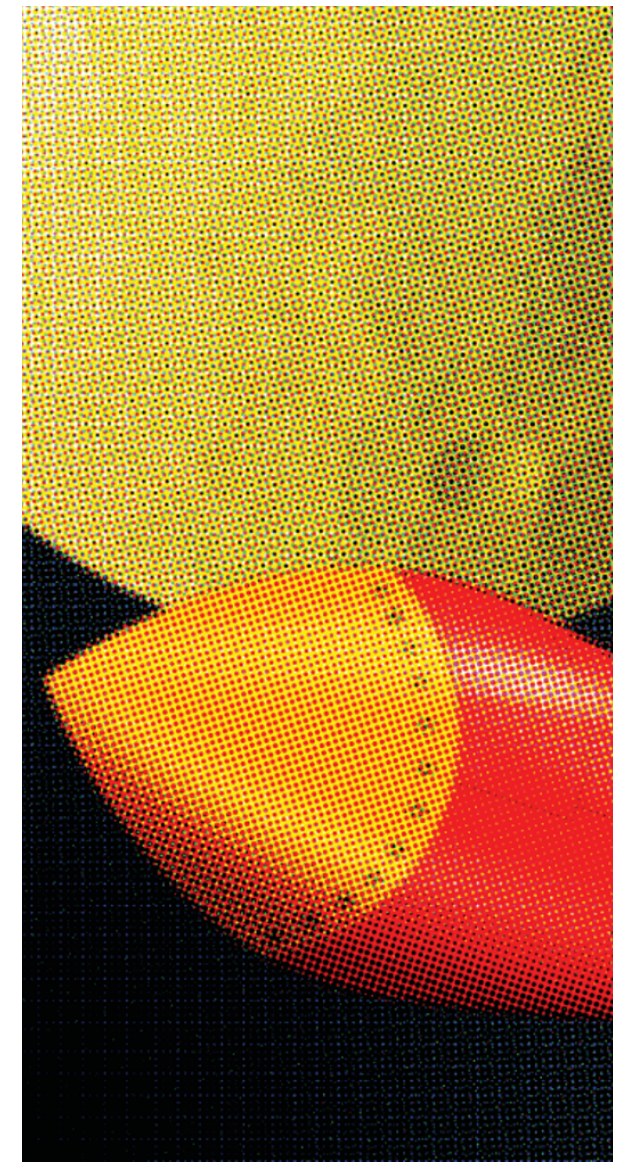

the $\mathrm{X}$ chromosome. So, although selection once again has a key role in causing this bias, in this case there is likely to be a purely mechanistic component to the bias.

So, it seems that selection primarily powers the genic traffic that

\section{TECHNOLOGY}

\section{A robot scientist}

Thanks to a new system developed by Ross King et al., scientists could soon be spending less time formulating and testing hypotheses and more time making "...the high-level creative leaps at which they excel".

King et al. have developed a 'robot scientist' that takes the integration of robotics and scientific discovery to a new level. It consists of a master computer that controls the system and carries out the scientific reasoning, a liquid-handling robot and a plate reader, along with their control computers. It runs software that includes background biological information, a logical inference engine and codes that generate hypotheses, select experiments and integrate the whole system.

Functional genomics was the testing ground for the robot scientist: specifically, dissection of the yeast aromatic amino acid (AAA)-synthesis pathway. First, the authors developed a 'logical formalism', which translates biological data into formulae for the computer. For the AAA pathway, data were taken from the Kyoto Encyclopedia of Genes and Genomes (KEGG). Using the logical formulae, Prolog, the robot scientist's logicprogramming language, then generated a model of the AAA pathway. Next, the robot scientist formulated hypotheses about the relationships between AAA-enzymatic reactions and open reading frames, devised and ran experiments to test them, interpreted the results to discount inconsistent hypotheses, and so on. The robot scientist essentially performed as well as human scientists, predicting at least $80 \%$ of all possible experiments.

Next, King et al. compared the accuracy (that is, the number of correct predictions made) versus the monetary cost of different experimental selection strategies. In the long term, the robot scientist's machine-learning system - active selection of experiments (ASE)-Progol — was more cost effective than choosing the cheapest experiment or a random experiment. 


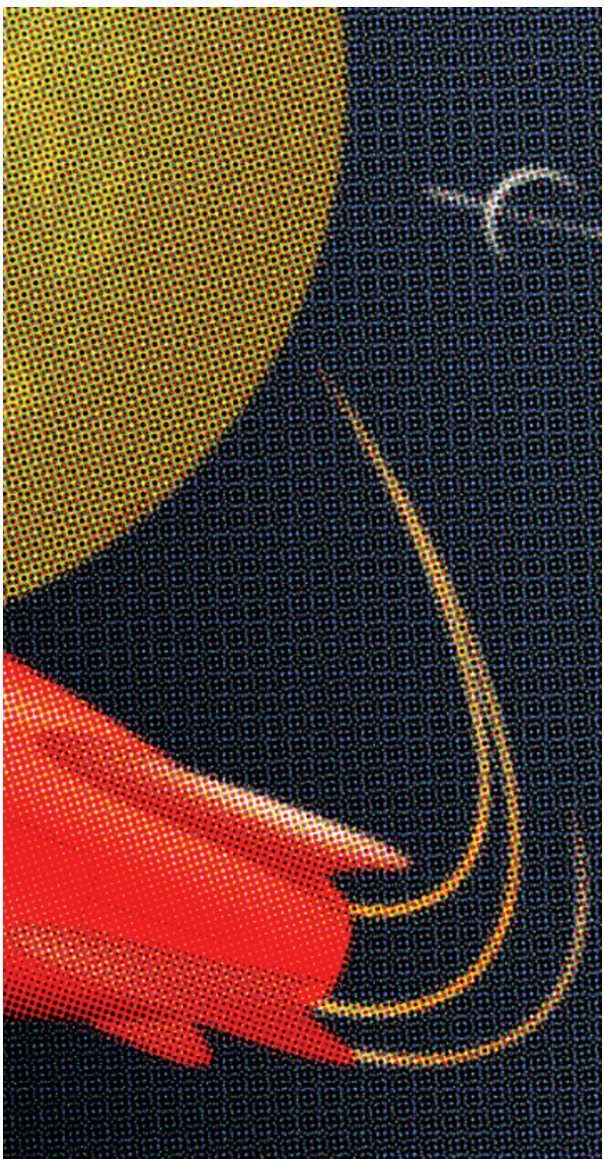

travels to and from the mammalian $\mathrm{X}$ chromosome. However, is the cement still wet on this busy genomic highway or is it a well-worn track? Emerson and colleagues answer this question with a comparative analysis of the mouse and human genomes,

However, before robot scientists appear in laboratories everywhere, there is still a lot of work to be done. The authors are now testing whether their system can uncover the role of genes for which no functional information is available. This will require the translation of many bioinformatic databases into logical formulae and the extension of their hypothesis-generation method. But it does seem that the potential of this system to be applied to many scientific problems will ensure that, one day, the use of robot scientists will be commonplace.

6) References and links ORIGINAL RESEARCH PAPER King, R. D. et al. Functional genomic hypothesis generation and experimentation by a robot scientist. Nature 427, 247-252 (2004) WEB SITES

ASE-Progol:

ftp://www.comp.rgu.ac.uk/pub/staff/chb/ systems/ase_progol/version_1.0 KEGG: http://www.genome.ad.jp/kegg which showed that most retrogenes that have escaped the $\mathrm{X}(12 / 15)$ or have moved to it $(10 / 13)$ did so before the mouse-human divergence. Clearly, the turnover of genes on the $\mathrm{X}$ chromosome is an ancient but ongoing process.

The fascinating picture of dynamic X-chromsome evolution that Emerson and colleagues have revealed invites a bit of genomic crystal-ball gazing. Is it just a matter of time before the X chromosome becomes the exclusive preserve of genes that are advantageous to males when hemizygous and that are silenced in female tissues, whereas all genes that are favourable to males when homozygous will be shifted to the autosomes?

$$
\text { Nick Campbell }
$$

\section{(1) References and links} ORIGINAL RESEARCH PAPER Emerson, J. J. et al. Extensive gene traffic on the mammalian X chromosome. Science 303, 537-540 (2004) FURTHER READING Long, M. et al. The origin of new genes: glimpses from the young and old. Nature Rev. Genet. 4, 865-875 (2003) | Betran, E. et al. Retroposed new genes out of the X in Drosophila. Genome Res. 12, 1854-1859 (2002) WEB SITES

Esther Betran's laboratory:

http://www3.uta.edu/faculty/betran Henrik Kaessmann's laboratory: http://www.unil.ch/cig/page6396_en.html Manyuan Long's laboratory: http://pondside.uchicago.edu/ حonglab/longlab.html

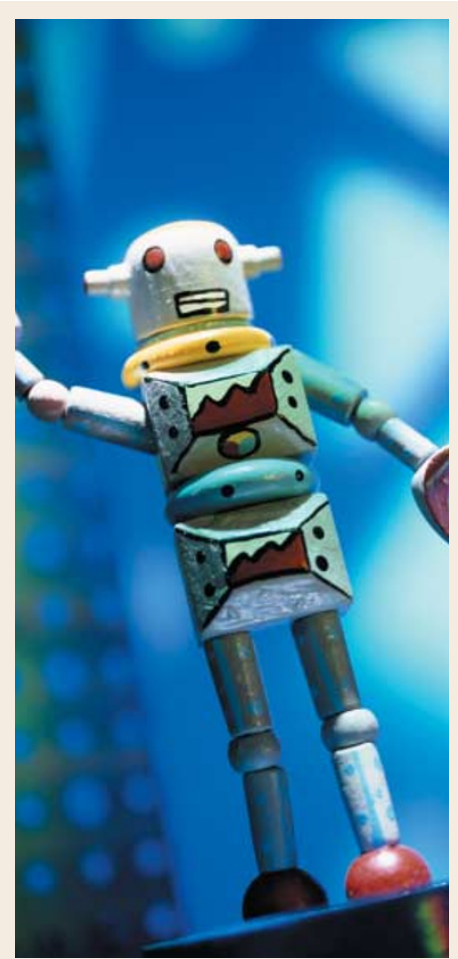

\section{IN BRIEF}

\section{DEVELOPMENTAL BIOLOGY}

fgfo mRNA decay establishes a gradient that couples axial elongation to patterning in the vertebrate embryo.

Dubrulle, J. \& Pourquié, O. Nature 427, 419-422 (2004)

Axial development in vertebrate embryos proceeds in a stereotypical manner whereby cells differentiate according to their position in a protein gradient. This paper shows how the Fgf8 gradient that controls this process might form in the chick. $f g f 8$ is transcribed only in tail-bud cells but this process stops as these cells move anteriorly during development. The protein gradient is consequently formed as the cells' supply of mRNA dwindles, therefore providing the answer to a long-standing question.

\section{POPULATION GENETICS}

\section{Evidence for extensive transmission distortion in the} human genome.

Zöllner, S. et al. Am. J. Hum. Genet. 74, 62-72 (2004)

Mendel's laws predict that a diploid organism should transmit each chromosome at a similar frequency. Deviations from this 1:1 ratioknown as segregation distortion - occur in many species and for various reasons. By examining genome data from 148 families, the authors conclude that segregation distortion is extensive in humans and that many loci underlie this effect.

\section{GENE REGULATION}

A noncoding RNA is required for the repression of RNApolll-dependent transcription in primordial germ cells.

Martinho, R. G. et al. Curr. Biol. 14, 159-165 (2004)

Unlike somatic cells, primordial germ cells (PGCs) - those that will develop into eggs and sperm - need to remain undifferentiated: they are thought to do so by inhibiting RNApolII transcription. Ruth Lehmann's group has now found that a non-coding RNA that is encoded by the polar granule component ( $p g c$ ) gene blocks RNApolII activity in PGCs, possibly by preventing transcription-activating enzymes from reaching the nucleus.

\section{MEDICAL GENETICS}

\section{Molecular and comparative genetics of mental retardation.}

Inlow, J. K. \& Restifo, L. L. Genetics (in the press)

Mental retardation (MR) is a common and genetically heterogeneous form of cognitive impairment. Jennifer Inlow and Linda Restifo estimate there to be hundreds of MR genes, 282 of which they have identified by data mining the Online Mendelian Inheritence in Man (OMIM) database and the literature. A total of $76 \%$ of these genes have functional orthologues in Drosophila, which indicates that this fly could be the ideal model to use to dissect the genetic basis of MR. 